\title{
The Interstellar Medium in the Magellanic Bridge
}

\author{
N. Lehner, F.P. Keenan, J.V. Smoker, P.L. Dufton, W.R.J. Rolleston, \\ F.C. McKenna \\ Department of Pure and Applied Physics, The Queen's University of \\ Belfast, Belfast, BT7 1NN, Northern Ireland
}

\author{
K.R. Sembach \\ The John Hopkins University, Department of Physics and Astronomy, \\ Bloomberg Center, 3400 N. Charles Street, Baltimore, MD 21218, USA
}

\section{J.E. Tohline}

Department of Physics and Astronomy, Louisiana State University, Baton Rouge, LA 70803-4001, USA

\author{
G. White, S. Stanimirović \\ Department of Physics, University of Western Sydney Nepean, \\ Kingswood, NSW, Australia
}

\begin{abstract}
HST ultra-violet and ground-based optical spectroscopy are presented for DI 1388, an early-type star in the Magellanic Bridge. These data have been supplemented with $\mathrm{H}$ I radio mapping of its environment. The spectroscopy shows interstellar components at LSR velocities of 0 , 90 and $200 \mathrm{~km} \mathrm{~s}^{-1}$ - corresponding to the local ISM, a high velocity cloud and the Magellanic Bridge ISM, respectively. The last is the first absorption line detection of this material and shows the presence of both low (e.g., N I) and high (Si IV) ionization material. Further optical and HST UV observations of DI 1338 and another Bridge star, DGIK 975, are scheduled to investigate the chemical homogeneity and star formation history of the Bridge.
\end{abstract}

\section{Introduction}

The Magellanic Bridge probably originated some $200 \mathrm{Myr}$ ago as a result of a tidal interaction during an LMC/SMC close approach, which resulted in material being pulled from the wing of the SMC. Detailed studies of the interstellar lines towards stars in the Bridge should give valuable information on its composition and origin.

Previously, we have obtained AAT spectra for five B-type stars in the Bridge (Hambly et al. 1994; Rolleston et al. 1998). Although these were principally used to measure stellar features, they also provided the first detection of interstellar absorption lines from the Bridge gas. Here we present HST UV spectroscopy 
and H I radio mapping of DI 1388 (RA: 0257 11.94, Dec: 025711.94 (J2000), $V=14.39, B-V=-0.26$, MK Type: B0V, $v \sin i=180 \pm 30 \mathrm{~km} \mathrm{~s}^{-1}$ ).

\section{Observations and Results}

HST Observations: STIS with the E140 grating provided wavelength coverage from 1150 to $1700 \AA$ at a resolution of $\sim 3 \mathrm{~km} \mathrm{~s}^{-1} \mathrm{pixel}^{-1}$ and $\mathrm{S} / \mathrm{N}$ of $\sim 10$. Absorption components with $v_{\mathrm{lsr}} \sim 0,90,200 \mathrm{~km} \mathrm{~s}^{-1}$ are present. The disk absorption lines are normally saturated, while the HVC absorption is weak and only a few chemical elements are identified. The Bridge absorption is detected unambiguously for 16 lines of Si II, Si III, Si IV, O I, S II, N I, C II, Fe II, Al II, Ni II.

ATCA Observations: Seven sightlines were observed, including one towards DI 1388. The peak brightness temperature of $11.2 \mathrm{~K}$ compares with the nearest single-dish Parkes values of $4 \mathrm{~K}$ (McGee \& Newton 1986). The $\mathrm{H}$ I column density map from the ATCA data has a maximum value of $\mathrm{n}_{H}=1 \times 10^{20} \mathrm{~cm}^{-2}$ compared with single-dish values of $\sim 5 \times 10^{20} \mathrm{~cm}^{-2}$. The ATCA maps show no correlation between peak $\mathrm{H}$ I temperatures and stellar positions.

\section{Discussion}

HST observations of a second Bridge star, DGIK 975, will be obtained next year, while AAT time has been allocated to re-observe both stars at high resolution and $\mathrm{S} / \mathrm{N}$. Our optical, UV, and new single-dish radio data will be combined to determine accurate physical parameters for the Bridge interstellar gas, including:

- Bridge origin from chemical composition: The Bridge composition should reflect that of the SMC. We will compare our Bridge abundances with those found in both the LMC and SMC and in the Magellanic Stream to investigate the proposition that both the Stream and Bridge originated from the same material.

- Star formation in the Bridge: Although the Bridge is believed to be approximately $200 \mathrm{Myr}$ old, our B-type targets have ages of $\leq 20 \mathrm{Myr}$. Hence star formation is still occurring in the Bridge. Given its very low $\mathrm{H}$ I column density, the mechanism is probably different from that operating in galactic disks. It has been suggested that collisions between cloudlets within HVCs in the halo of our Galaxy leads to star formation. Given that the H I densities inferred for the Bridge and the HVCs are similar, such a formation mechanism may be operating in the Bridge. We therefore intend to compare our Bridge results with those found for halo sightlines.

\section{References}

Hambly N.C., Dufton P.L., Rolleston W.R.J., et al. 1994, A\&A, 285, 716

McGee R.X., \& Newton L.M. 1986, PASA, 6, 471

Rolleston W.R.J., Dufton P.L., Mc Erlean N.D., et al. 1999, A\&A, submitted 